\title{
Particle tracking in the CALET experiment
}

\author{
Paolo Maestro* (for the CALET Collaboration) \\ Department of Physical Sciences, Earth and Environment \\ University of Siena, via Roma 56, 53100 Siena (Italy) \\ E-mail: paolo.maestro@pi.infn.it
}

\author{
Nicola Mori \\ INFN sezione di Firenze \\ via B. Rossi 1, 50019 Sesto Fiorentino (Italy) \\ E-mail: moriefi.infn.it
}

The Calorimetric Electron Telescope (CALET) is a space mission installed on the Exposed Facility of the Japanese Experiment Module (JEM-EF) of the International Space Station (ISS) in August 2015 and collecting data since October 2015. In addition to high precision measurements of the electron spectrum up to TeV scale, CALET will also investigate the mechanism of cosmic-ray (CR) acceleration and propagation in the Galaxy, by performing direct measurements of the energy spectra and elemental composition of $\mathrm{CR}$ nuclei from $\mathrm{H}$ to $\mathrm{Fe}$, and the abundance of trans-iron elements up to about $\mathrm{Z}=40$. The instrument consists of two layers of segmented plastic scintillators to identify the particle charge, a thin (3 radiation lengths) tungsten-scintillating fiber calorimeter providing accurate particle tracking, and a thick (27 radiation lengths) calorimeter made of lead-tungstate crystal logs. In this paper we will describe an original reconstruction method of the primary particle direction based on a combinatorial Kalman filter algorithm. This method exploits the fine granularity and imaging capability of the IMC and provides robust track finding and fitting, allowing to identify the incident CR track in a large amount of shower particle tracks backscattered from the calorimeter. The track fitting algorithm has been extensively validated and tuned with simulated data. Its performance (angular resolution, impact point resolution, tracking efficiency) for electrons and nuclei will be discussed and comparisons between flight data and simulations will be shown.

35th International Cosmic Ray Conference - ICRC2017-

10-20 July, 2017

Bexco, Busan, Korea

* Speaker. 


\section{Introduction}

The CALET mission is a space experiment [1], funded by the Japanese AeroSpace Agency (JAXA), the Italian Space Agency (ASI) and NASA. CALET was launched on August $19^{\text {th }}, 2015$ by the Japanese rocket H-II Transfer Vehicle (HTV-5) and robotically installed on the Japanese Experiment Module Exposure Facility (JEM-EF) of the ISS on August $24^{\text {th }}$, for a two-year mission, with a possible first extension to 5 years [1]. The on-orbit commissioning phase aboard the ISS was successfully completed in the first days of October 2015, without remarkable issues, and since then it is taking science data continuously.

The main scientific goal is to search for possible clues of the presence of astrophysical sources of high-energy electrons nearby the Earth or signatures of Dark Matter, by measuring accurately the $e^{-}+e^{+}$spectrum from $1 \mathrm{GeV}$ up to several TeV. CALET will also also investigate the mechanism of cosmic-ray (CR) acceleration and propagation in the Galaxy, by performing direct measurements of the energy spectra and elemental composition of $\mathrm{CR}$ nuclei from $\mathrm{H}$ to $\mathrm{Fe}$ up to hundreds of $\mathrm{TeV}$, and of the abundance of ultra-heavy Galactic CRs at few GeV/amu up to about $\mathrm{Z}=40$.

The CALET instrument consists of three detectors: a CHarge Detector (CHD), a finely segmented pre-shower IMaging Calorimeter (IMC), and a Total AbSorption Calorimeter (TASC). The TASC is a homogeneous calorimeter made of 192 PWO "logs" $\left(2 \times 1.9 \times 32 \mathrm{~cm}^{3}\right)$ arranged in 12 layers, with a total thickness equivalent to 27 radiation lengths $\left(\mathrm{X}_{0}\right)$. The logs in the top layer are readout by photomultiplier tubes (PMTs), while a dual photodiode/avalanche-photodiode system is used for the readout of the remaining layers. The TASC was designed to determine the energy of the incident electrons and gamma rays with an excellent energy resolution, better than $2 \%$ above $100 \mathrm{GeV}$, and provide a high discrimination between electromagnetic (e.m.) and hadronic cascades. The IMC consists of 7 tungsten plates interleaved with double layers of $1 \mathrm{~mm}^{2}$ scintillating fibers (SciFi), arranged in belts along orthogonal directions and readout by multianode PMTs, capped by an additional SciFi layer pair. Its fine granularity allows to accurately image the longitudinal and lateral profiles of the initial shower development, enhancing the electron-proton discrimination and providing electron-gamma separation. Moreover it provides accurate particle tracking and CR identification by multiple $\mathrm{dE} / \mathrm{dx}$ sampling. The total thickness of the instrument is equivalent to 30 $X_{0}$ and 1.3 proton interaction lengths $\left(\lambda_{I}\right)$. The geometrical factor is $0.12 \mathrm{~m}^{2} \mathrm{sr}$ and the total weight is $613 \mathrm{~kg}$. Calibration and test of the instrument took place at CERN SPS during five campaigns between 2010 and 2015 with beams of electrons, protons and relativistic ions.

\section{Particle tracking with IMC}

Track finding and reconstruction is of utmost importance for CALET. An accurate particle tracking is necessary to:

- identify the CHD paddles and IMC fibers crossed by an incoming CR particle in order to measure its charge and the point where it starts showering;

- compute topological calorimetric variables, like the width of the shower with respect to the particle trajectory, which are used for proton rejection in electron analysis. 
These tasks can be really complicated by the large amount of shower particle tracks backscattered from the TASC and delta rays along the incident particle track, which generate many "hits" in IMC. These hits must be grouped into track candidates and subsequently fitted with a track model to find their parameters. High hit multiplicity can generate multiple track candidates and increase the computation time; electronic noise generating spurious hits might worsen the situation. On the other hand, eventual detector inefficiencies might remove some hits generated by physical particles, thus making it harder to identify the real physical tracks.

This method exploits the fine granularity and imaging capability of the IMC, and is based on the combinatorial Kalman filter algorithm to provide robust track finding and fitting [2]. This is a hybrid technique of data analysis for simultaneous track and vertex fitting widely used in highenergy experiments at the accelerators [3], but not applied so far in a calorimetric CR experiment. It can easily handle a high multiplicity of hits, as expected in the IMC for high-energy shower events, and reconstruct multiple track candidates, with a fast computation time, even in case of detector inefficiencies or dead channels.

\subsection{Tracking algorithm}

The track finding problem in CALET can be decomposed in two independent two-dimensional problems in the $\mathrm{XZ}$ and $\mathrm{YZ}$ views, respectively, since IMC SciFi layers are oriented only in either $\mathrm{X}$ or $\mathrm{Y}$ direction, and placed at a different $\mathrm{Z}$ coordinates. Two straight lines in orthogonal planes crossing the IMC layers at various positions represent the projections of the particle trajectory. Neighboring hits in each IMC layer are clustered, and the cluster positions (computed as the centerof-gravities (COG) of the fibers belonging to each cluster) are taken as candidate track points for the combinatorial Kalman filter. Track finding begins from the upper IMC layer. A candidate track is created for each possible combination of clusters in the first two layers, including tracks with a missing hit. Each track candidate is fitted to a straight line and evolved separately, i.e. its impinging angle and impact point (forming the so-called "status vector") on the next layer $(k+1)$ are estimated using only the information (status vector and covariance matrix) at the previous layer $k$. This predicted state can be used to identify the cluster to be assigned to the track at layer $k+1$ : a possible criterion is to associate the cluster nearest to the predicted measurement. However that does not provide a robust algorithm, i.e., an algorithm that is resilient to wrong initial assumptions. If the initial track estimate is wrong, then a wrong measurement might be associated to the track, like a noise cluster or a fluctuating measurement belonging to another track. The combinatorial formulation of the Kalman filter deals with this problem by creating a new track candidate for each cluster on layer $k+1$, which lies sufficiently near to the predicted state, i.e. within the position error computed for the cluster. Then the candidate track is "branched" into many candidates, and each of them can be evolved separately. For each new branch, information about the track point in layer $k+1$ is then combined with the predicted state to produce a "filtered" estimate of the status vector and the covariance matrix at layer $k+1$. The residual of the filtered estimate of the measurement with respect to the true cluster position can then be computed and from that the $\chi^{2}$ of the fitted track can be updated. The procedure is then iterated to $k+2$-th layer and so on. Obviously such combinatorial algorithm can produce a very large number of track candidates, which can considerably slow down the computation. To cope with this, a candidate track may be discarded if, at any point of its evolution, it has a $\chi^{2}$ greater than a predefined value (typically 
10) or a too large number of missing points $(>2)$. Candidate tracks reaching the last point are "smoothed", i.e. the information given by the full measurements set is back-propagated to all the previous points. The smoothed state on first layer will give the best estimate of the impact angle and point on the first detector layer of the particle track.

For high-energy shower events triggering the instrument, in order to reduce the tracks combinations and then the processing time, only IMC clusters within a ROI (region-of-interest) are fed into the algorithm. The ROI include all the SciFi's within $\pm 5 \mathrm{~cm}$ from the intercept of the ROI axis with each IMC layer. Different definitions of the ROI axis are implemented depending on the event topology in IMC. If, in each of the last four IMC layers, there is a SciFi cluster containing more than half the energy deposited in the layer, then ROI axis is defined as the straight line fitted to the COG of these four clusters and the most energetic TASC log of the first TASC layer. This condition is effective to identify a rough direction for CR particle, like electrons, interacting early and developing a well defined shower in IMC. Instead, if the above criterion is not verified, the shower axis, which is fitted to the COG of the clusters of TASC "logs" inside the shower core, is back-projected to define the ROI in IMC.

Among all the tracks found by the combinatorial Kalman Filter algorithm, a candidate primary track has to be identified. First, equivalent tracks are compared. Two tracks are defined to be equivalent if on any given plane they are associated to the same hit, neglecting all the layers where there is no associated hit for at least one track. For each set of equivalent tracks, the one with the lowest $\chi^{2}$ is retained, and the others are discarded.

Among non-equivalent tracks, the one passing closer to the core of the shower reconstructed in TASC is chosen as the primary particle track.

\section{Tracking performance}

Since the IMC tracking performance depends on the incident particle species and energy, the algorithm was tuned and validated with different samples of particles simulated by the Monte Carlo package FLUKA [4, 5]. Electrons, protons and He, C, Fe nuclei were generated isotropically in direction and according to a spectrum covering energies from $10 \mathrm{GeV}$ to $200 \mathrm{TeV}$ for nuclei and $20 \mathrm{TeV}$ for electrons, following a power law in energy $E^{-1}$. The primary CALET mission trigger, called High-Energy Trigger (HET), was modelled in simulation. It is based on the coincidence of a signal from the top layer of the TASC and the sum of signals from the four last layers of SciFi belts in IMC, with thresholds chosen to ensure 95\% efficiency for electrons above $10 \mathrm{GeV}$. Simulated particles generating an HET event are traced, and sample of particles inside the CALET acceptance (i.e. with a reconstructed track length $>27 X_{0}$ and entrance point in the instrument above the fifth IMC layer) are used to evaluate the tracking performance in terms of spatial and angular resolution, and tracking efficiency.

To estimate the angular resolution, the true MC generated direction of the particle (defined by the incident angles $\theta_{x}^{M C}$ and $\theta_{y}^{M C}$ in $\mathrm{XZ}$ and $\mathrm{YZ}$ views, respectively) is compared with the reconstructed information $\left(\theta_{x, y}^{r e c}\right)$, as shown for electrons in the top left panel of Fig. 1. A Point Spread Function (PSF) is calculated as the half-width of the interval centered on the mean value of the residual distribution in each view (i.e. $\Delta \theta_{x, y}=\theta_{x, y}^{M C}-\theta_{x, y}^{r e c}$ ) and containing $68.3 \%$ of the events (Fig. 1, top right panel). 

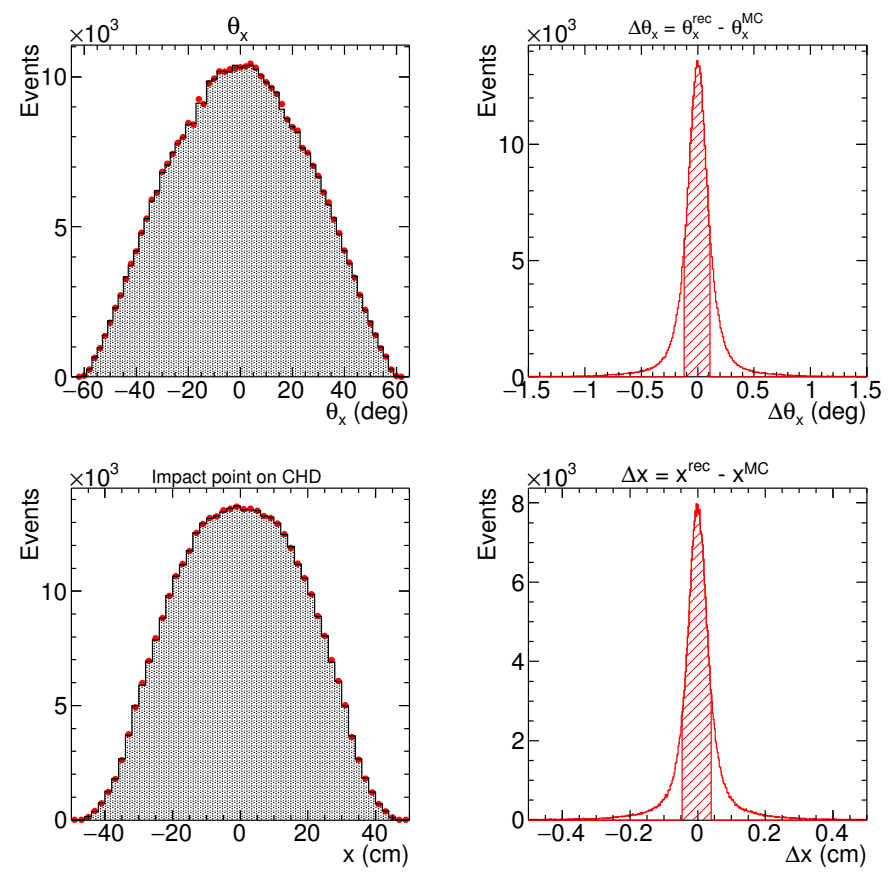

Figure 1: (Top left) Reconstructed incident angle in XZ view (red dots) superimposed to the corresponding MC generated angle, for electrons. (Top right) Residuals between the MC generated and the reconstructed angle in XZ view. The filled area centered on the mean value of the distribution contains $68.3 \%$ of the reconstructed events. Its half-width of $0.11^{\circ}$ is an estimate of the angular resolution of the tracking algorithm for electrons. (Bottom left) Electron $x$ coordinate of the incident point on top of the CHD. The filled histogram and the red dots represent the true impact point and the reconstructed one, respectively. (Bottom right) Residuals between the MC generated and the reconstructed $x$ impact point. The filled area centered on the mean value of the distribution contains $68.3 \%$ of the reconstructed events. Its half-width of $420 \mu \mathrm{m}$ is an estimate of the spatial resolution of the tracking algorithm for electrons.

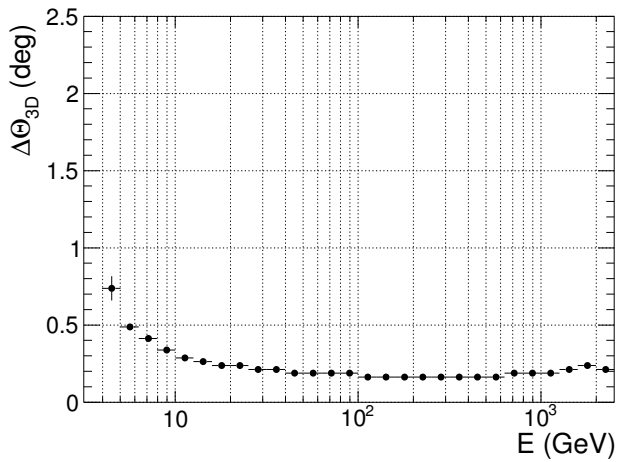

(a)

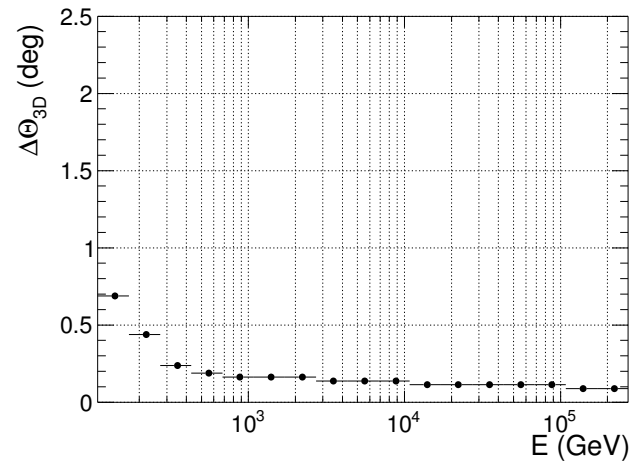

(b)

Figure 2: Resolution of the reconstructed arrival direction of the particle as a function of energy for electrons (a) and Fe nuclei $(b)$. 
In a similar way, an estimate of the spatial resolution at the particle incidence point on top of the CHD is performed. The residuals for the $x$ and $y$ coordinates are calculated as the distance between the true position $\left(x_{M C}, y_{M C}\right)$ of the incident particle at $z=0$ (i.e. top of CHD) and the one extrapolated from the reconstructed track $\left(x_{r e c}, y_{r e c}\right)$. In order to quote a $\sigma$ value for the resolution, a $68.3 \%$ interval around the mean is defined, in analogy with the PSF (Fig. 1, bottom panels).

For simulated nuclei events, the plots of these quantities are not shown for brevity, but have a similar behaviour, albeit the performances are different. The PSF of $\theta_{x}$ is: $0.1^{\circ}$ for electrons, $0.55^{\circ}$ for protons, $0.45^{\circ}$ for $\mathrm{He}, 0.22^{\circ}$ for $\mathrm{C}, 0.09^{\circ}$ for $\mathrm{Fe}$. The $\mathrm{CHD}$ impact point resolution is: $420 \mu \mathrm{m}$ for electrons, $1.5 \mathrm{~mm}$ for protons, $1.2 \mathrm{~mm}$ for $\mathrm{He}, 580 \mu \mathrm{m}$ for $\mathrm{C}, 260 \mu \mathrm{m}$ for Fe.

The angular informations of $\mathrm{XZ}$ and $\mathrm{YZ}$ views can be combined to determine the parameters $(\theta, \phi)$ of the arrival direction of the particle in the space. The $3 \mathrm{D}$ angular resolution $\Delta \Theta_{3 D}$ is quoted as the PSF of the distribution of the scalar product between the reconstructed and the true $\mathrm{MC}$ arrival direction. The angular resolution as a function of the particle energy is shown for electrons and Fe nuclei in Fig. 2. $\Delta \Theta_{3 D}$ is $\sim 0.2^{\circ}$ for electrons between $15 \mathrm{GeV}$ and $2 \mathrm{TeV}$; for Fe nuclei, $\Delta \Theta_{3 D}$ is $\sim 0.2^{\circ}$ at $1 \mathrm{TeV}$, and it goes down to $0.1^{\circ}$ above $10 \mathrm{TeV}$ particle energy.

The tracking efficiency was calculated taking into account the whole acceptance of the CALET telescope, resulting into an estimated efficiency of $>95 \%$ for simulated electrons above $10 \mathrm{GeV}$, $>90 \%$ for protons and $\mathrm{He}$ nuclei, and $>95 \%$ for heavier nuclei above $100 \mathrm{GeV}$ particle energy.

\section{Tracking with flight data}

In order to validate the tracking procedure with real data, the algorithm is applied to samples of proton and carbon nuclei selected using the first few month of data collected by the CALET experiment on the ISS. Particles generating High-Energy Trigger events are required to have an associated reconstructed track with $\chi^{2}<5$ and more than 4 track points in each view. Particles crossing the whole instrument from top of CHD to the bottom of TASC are selected, and signals of CHD paddles crossed by the track are used to identify the CR particle charge. Events with a total energy deposited in TASC larger than $100 \mathrm{GeV}$ for protons and $45 \mathrm{GeV}$ for $\mathrm{C}$ nuclei are selected.

The reconstructed incident angle in both views and the impact point on top of CHD are compared with the corresponding simulated distributions. In order to compare with flight data, MC events, which were generating according to a spectrum $E^{-1}$, are reweighted to a spectrum $E^{-\gamma}$, with $\gamma=-2.65$ for $\mathrm{C}$ nuclei and -2.75 for protons, respectively.

A good agreemeent between data and simulation is found as shown in Figs. 3, 4, and 5.

\section{Conclusion}

An algorithm based on a combinatorial Kalman filter has been developed to track charged CR particles in CALET. The method has been tuned and validated with simulated samples of electrons and nuclei from $\mathrm{H}$ up to $\mathrm{Fe}$ between tens of $\mathrm{GeV}$ and the multi-TeV scale. It turned out to be effective in identifying with high efficiency the arrival direction of primary CR's in a huge background of secondary tracks produced by shower particles in the calorimeter. The angular and spatial resolution achieved with this method are excellent for a calorimetric instrument, allowing a precise determination of the CHD paddles and Scifi crossed by the incoming particle necessary 

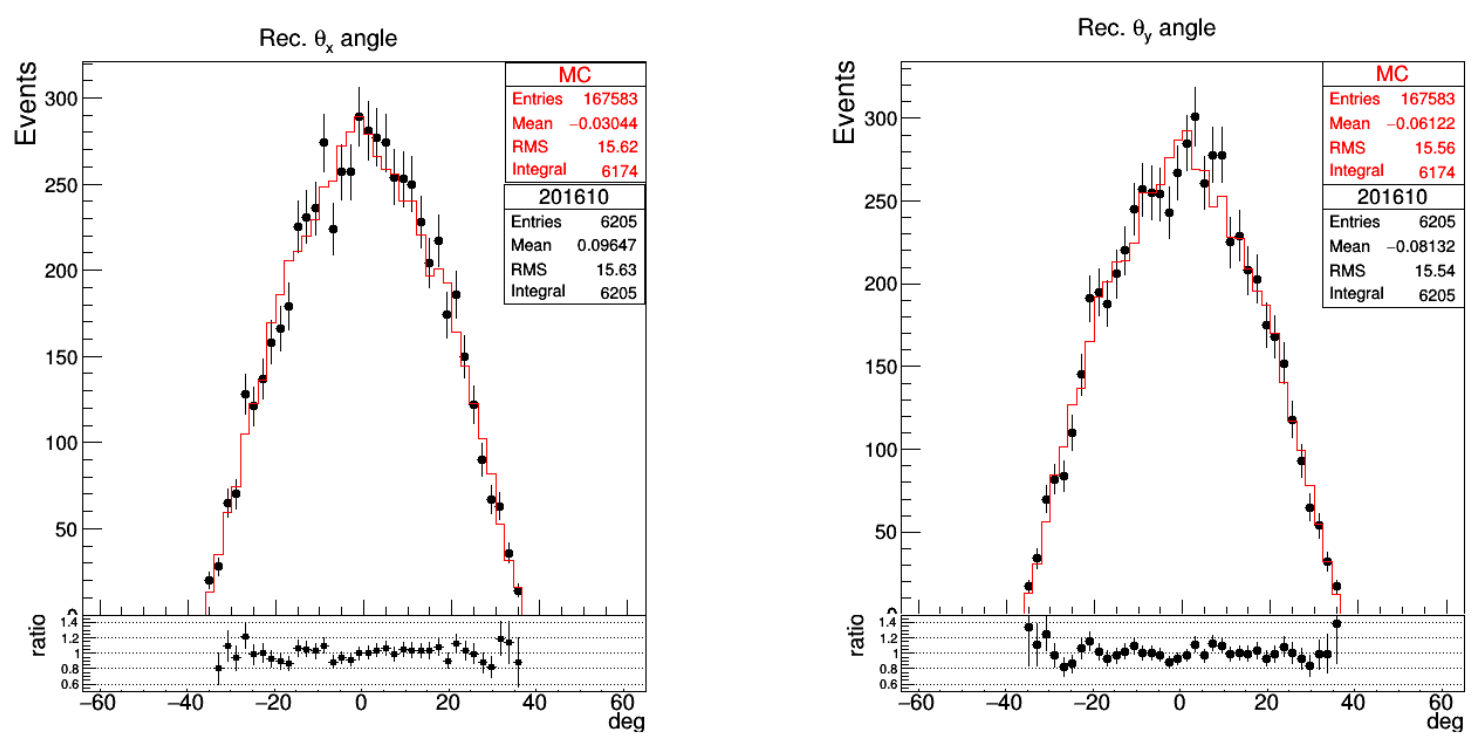

Figure 3: Reconstructed incident angles $\left(\theta_{x}, \theta_{y}\right)$ in $\mathrm{XZ}$ and $\mathrm{YZ}$ views (black dots) for a sample of protons selected in one month of flight data, superimposed on the corresponding simulated distributions (red histograms). The ratio between flight data and MC distributions is shown in the bottom panel.

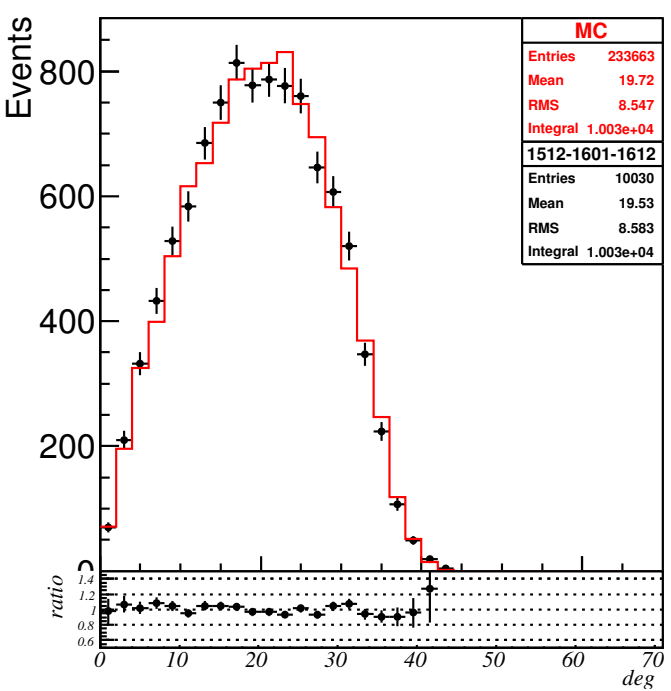

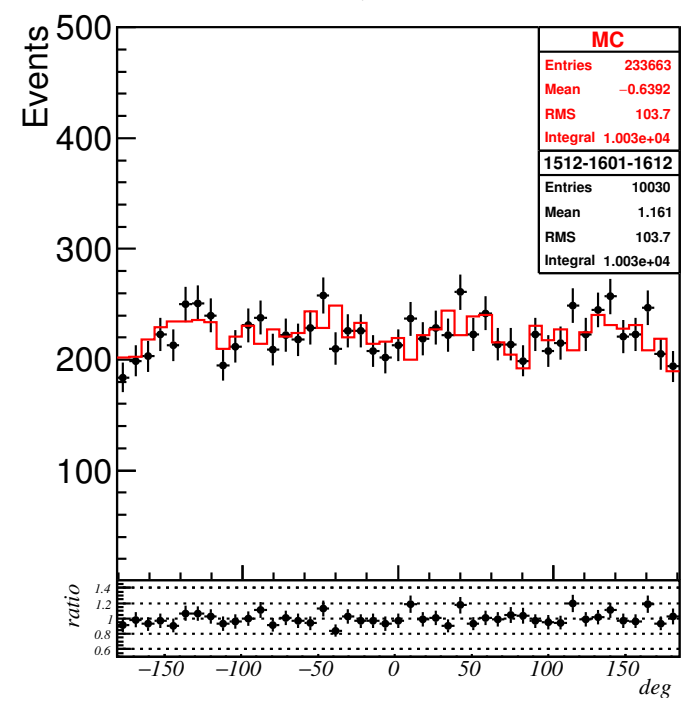

Figure 4: Reconstructed $\theta$ and $\phi$ angles (black dots) for a sample of carbon nuclei selected in three months of flight data, superimposed on the corresponding simulated distributions (red histograms). The ratio between flight data and MC distributions is shown in the bottom panel. 

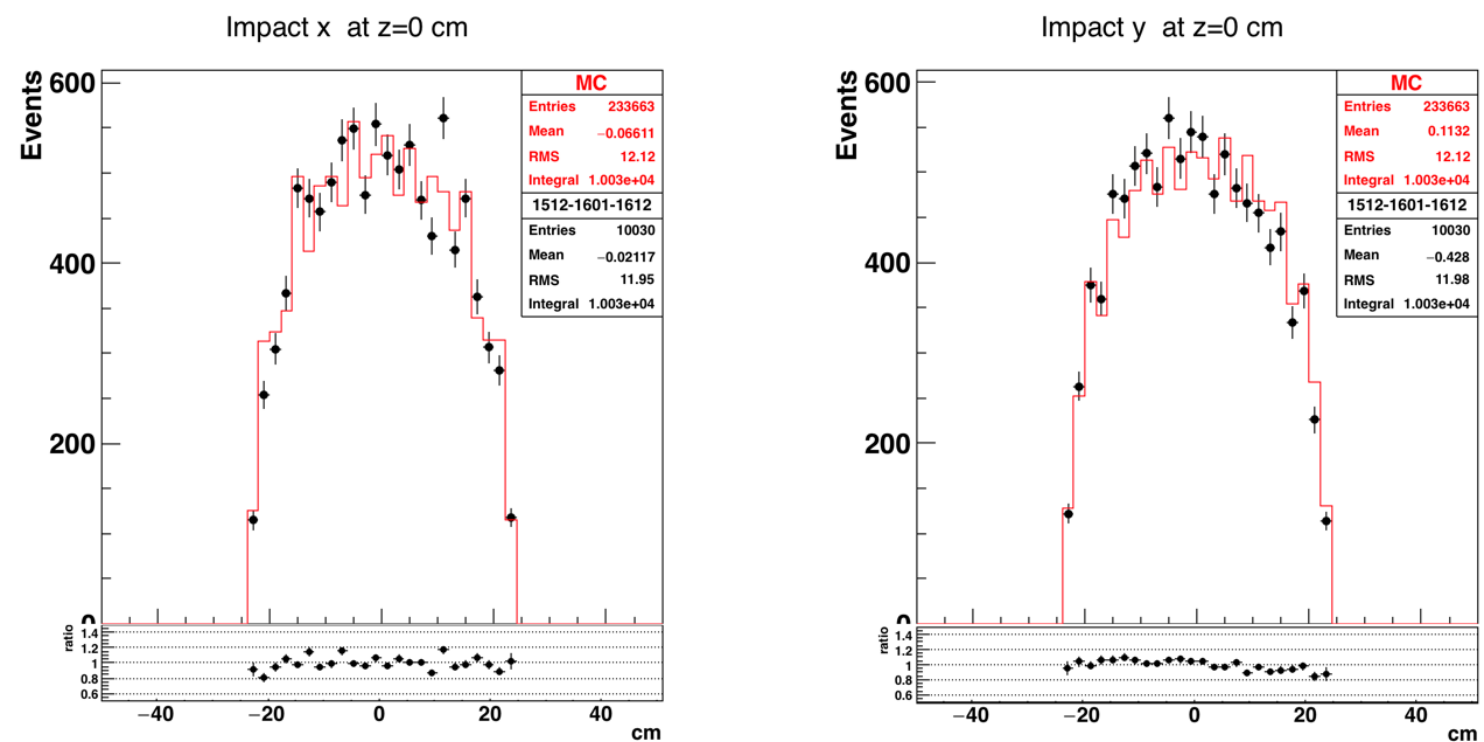

Figure 5: Reconstructed impact point on top of CHD (black dots) for a sample of carbon nuclei selected in three months of flight data, superimposed on the corresponding simulated distributions (red histograms). The ratio between flight data and MC distributions is shown in the bottom panel.

for its identification, and an accurate definition of the geometrical factors for flux measurements. Comparison of the reconstructed tracking information shows a good agreement between samples of flight and simulated data, demonstrating the reliability of the method and good modeling and simulation of the instrument.

\section{References}

[1] S. Torii, Pos(ICRC2015) 581

[2] R. Frühwirth, Nucl. Instr. and Meth. A, 262 (1987), 444-450

[3] A. Strandlie and R. Frühwirth, Rev. Mod. Phys., 82 (2010), 1419-1458

[4] A. Ferrari, P.R. Sala, A. Fassó, and J. Ranft, CERN-2005-10 (2005), INFN/TC_05/11, SLAC-R-773

[5] T.T. Böhlen et al., Nuclear Data Sheets 120, 211-214 (2014) 